\title{
Integrated analysis of microRNA and transcription factor reveals important regulators and regulatory motifs in adult B-cell acute lymphoblastic leukemia
}

\author{
XIAO-CONG LIN ${ }^{1,2}$, XIN-GUANG LIU ${ }^{1,2}$, YU-MING ZHANG ${ }^{1,3}$, NING LI ${ }^{1,3}$, ZHI-GANG YANG $^{1,3}$, \\ WEI-YU FU ${ }^{1,2}$, LIU-BO LAN ${ }^{1,2}$, HAI-TAO ZHANG ${ }^{1,2}$ and YONG DAI ${ }^{4}$
${ }^{1}$ Guangdong Provincial Key Laboratory of Medical Molecular Diagnostics, Guangdong Medical University, Dongguan, Guangdong 523808; ${ }^{2}$ Institute of Biochemistry and Molecular Biology, Guangdong Medical University, Zhanjiang, Guangdong 524023; ${ }^{3}$ Department of Hematology, Affiliated Hospital of Guangdong Medical University, Zhanjiang, Guangdong 524001; ${ }^{4}$ Clinical Medical Research Center, Shenzhen People's Hospital, Shenzhen, Guangdong 518020, P.R. China

Received November 8, 2016; Accepted December 16, 2016

DOI: $10.3892 /$ ijo.2016.3832

\begin{abstract}
B-cell acute lymphoblastic leukemia (B-ALL) is an aggressive hematological malignancy and a leading cause of cancer-related mortality in children and young adults. The molecular mechanisms involved in the regulation of its gene expression has yet to be fully elucidated. In the present study, we performed large scale expression profiling of microRNA (miRNA) and transcription factor (TF) by Illumina deep-sequencing and TF array technology, respectively, and identified 291 differentially expressed miRNAs and 201 differentially expressed TFs in adult B-ALL samples relative to their controls. After integrating expression profile data with computational prediction of miRNA and TF targets from different databases, we construct a comprehensive miRNA-TF regulatory network specifically for adult B-ALL. Network function analysis revealed 25 significantly enriched pathways, four pathways are well-known to be involved in B-ALL, such as PI3K-Akt signaling pathway, Jak-STAT signaling pathway,
\end{abstract}

Correspondence to: Professor Yong Dai, Clinical Medical Research Center, Shenzhen People's Hospital, 1017 Dongmen North Road, Shenzhen, Guangdong 518020, P.R. China

E-mail: daiyong22@aliyun.com

Abbreviations: B-ALL, B-cell acute lymphoblastic leukemia; ALL, acute lymphoblastic leukemia; T-ALL, T-cell acute lymphoblastic leukemia; miRNA, microRNA; TF, transcription factor; TFBS, transcription factor binding site; FBL, feedback loop; FFL, feed-forward loop; WHO, World health Organization; HRP, horseradish peroxidase; qPCR, quantitative PCR; TSS, transcription start site; GO, Gene Oncology; KEGG, Kyoto Encydopedia of Gene and Genomes; STAT5, signal transducer and activator of transcription 5; FLT3, FMS-like tyrosine kinase 3; AML, acute myeloid leukemia

Key words: adult B-cell acute lymphoblastic leukemia, microRNA, transcription factor, sequencing, microarray
Ras signaling pathway and cell cycle pathway. By analyzing the network topology, we identified 28 hub miRNAs and 19 hub TFs in the network, and found nine potential B-ALL regulators among these hub nodes. We also constructed a Jak-STAT signaling sub-network for B-ALL. Based on the sub-network analysis and literature survey, we proposed a cellular model to discuss MYC/miR-15a-5p/FLT3 feed-forward loop (FFL) with Jak-STAT signaling pathway in B-ALL. These findings enhance our understanding of this disease at the molecular level, as well as provide putative therapeutic targets for B-ALL.

\section{Introduction}

B-cell acute lymphoblastic leukemia (B-ALL) is an aggressive hematological malignancy characterized by uncontrolled and rapid proliferation of B-lymphoid precursor cells within the bone marrow $(1,2)$. B-ALL represents the most common type of ALL and accounts for $\sim 75$ and $85 \%$ of adult and childhood ALL cases, respectively $(3,4)$. It is estimated that nearly 4,000 de novo cases are diagnosed each year in the US (5). Although advances in risk-adapted intensive chemotherapy have dramatically improved the disease outcomes of childhood patients with B-ALL, this approach is less effective in adults, with a disease-related mortality of $\sim 60 \%(6,7)$. Relapse occur frequently following remission induction chemotherapy, and the expected survival of relapsed patients was less than 6 months $(8,9)$. Especially, the 5-year survival rate for adult patients is a dismal $7 \%$ at time of relapse (10). Chemotherapy-refractory and relapsed B-ALL remains the primary cause of cancer-related mortality in children and young adults $(5,11,12)$. Therefore, there is an urgent need to discover new therapeutic targets for B-ALL. Understanding the critical factors and molecular mechanisms associated with B-ALL initiation and progression is essential for further advances in therapy. Currently, many cytogenetic and genomic lesions have been shown to play important roles in the pathogenesis of B-ALL, which include hyperdiploidy $(11,13,14)$, hypodiploidy $(6,13,14)$, rearrangements of TEL, TCF3, MLL, BCR/ABL 
and $\operatorname{Ig} \mathrm{H}^{@}$ gene $(6,12,14,15)$, mutations of PAX5, CRLF2, IKZF1 and JAK gene $(6,11,15)$, deletion of CDKN2A/B (12). These aberrations are strongly correlated with disease characteristics, patient prognosis and the response to chemotherapy, however, understanding of the molecular mechanisms of this disease remains limited. In particular, the regulatory networks of B-ALL genes expression are still unclear.

miRNAs are small noncoding RNAs of $\sim 18$ to 25 nucleotides that inhibit gene expression at the post-transcriptional level through binding to the 3'-UTR of target mRNAs (16). A growing body of research evidence has confirmed the crucial roles of miRNA in various types of human cancers, including B-ALL (17). It has been reported that miR-125b-5p was upregulated in patients with B-ALL carrying the $\mathrm{t}(11 ; 14)(\mathrm{q} 24 ; \mathrm{q} 32)$ translocation. Ectopic expression of miR-125b-5p would induce hematopoietic fetal liver cells progresses to B-ALL, T-cell acute lymphoblastic leukemia (T-ALL) or a myeloproliferative neoplasm in xenograft mouse models (18). Furthermore, elevated levels of miR-125b-5p, miR-99a and miR-100 were correlated with resistance to daunorubicin and vincristine, while decreased expression of miR-708 was related to resistance to glucocorticoids in pediatric B-ALL $(16,17)$. miR-17-92 was significantly downregulated in the BCR-ABL-positive human B-ALL samples and its overexpression induced apoptosis of B-ALL cells in a BCR-ABL-dependent manner by targeting Bcl-2 (19).

Transcription factors (TFs) are protein factors that regulate gene transcription by specifically binding to cis-regulatory elements located in the promoter regions of target genes, which we now call transcription factor binding site (TFBS) (20). As key regulators of gene expression in organisms, TFs and miRNAs are able to mutually regulate one another in forms of feedback loops (FBLs) or cooperatively regulate the same target genes in a combinatorial manner to form feed-forward loops (FFLs) hence ensuring the precise and accurate control of gene expression (20,21). Recent investigations concerning the gene expression regulation by miRNA and TF in leukemia have provided new insights into disease pathogenesis (21-23). miR-223 and two TFs (C/EBP $\alpha$ and NFI-A) formed a regulatory circuitry to control the differentiation of acute promyelocytic leukemia cells (21). miR-196b can exert its effects by targeting the TF MYC and induce cell apoptosis in B-ALL (22). miR-19 was overexpressed in T-ALL cell lines and patients. Ectopic expression of miR-19 sustained activation of NF- $\kappa$ B pathway in T-ALL involving a feed-forward circuitry in miR-19, CYLD and $\mathrm{TF} N F-\kappa \mathrm{B}(23)$. However, global regulation of miRNAand TF-mediated control of gene expression in adult B-ALL is still poorly understood. Thus, an integrated analysis of miRNAs and TFs, as their cooperative interaction relates to B-ALL pathogenesis, requires examination.

In this study, we applied Illumina deep-sequencing and TF array technology to analyze the miRNA and TF expression profiles, respectively, in bone marrow samples of adult B-ALL patients and their controls. Based on a combined bioinformatics approach for prediction of miRNA and TF targets, we integrated differentially expressed miRNAs and TFs as well as B-ALL candidate genes to construct a B-ALL-related miRNA and TF regulatory network. After analyzing the network function and topology, we found some B-ALL-related pathways and hub regulators from the network, and further investigated their regulation properties in the subnetwork. This study will be helpful to decipher the complex molecular mechanisms underlying B-ALL, as well as to provide potential therapeutic targets for B-ALL in the future.

\section{Materials and methods}

Patient samples. A total of 25 bone marrow samples, including 15 patients derived from newly diagnosed and untreated adult B-ALL (7 males and 8 females; 18-71 years old; average age, 36.5 years) and 10 controls ( 6 males and 4 females; 10-73 years old; average age, 39.0 years), were used in this study. All samples were collected at the Affiliated Hospital of Guangdong Medical University (Zhanjiang, China). Diagnosis of B-ALL was determined according to the accepted WHO criteria (24-26). Control samples were obtained from 10 patients with unexplained fever or anemia whose bone marrow aspirates was demonstrated to be normal bone marrow morphology. All patients gave informed consent for participation in this study according to the Helsinki convention criteria, and the study was approved by the Ethics Committee of Affiliated Hospital of Guangdong Medical University.

Total RNA isolation. Total RNA was isolated using RNeasy kit (Qiagen, Hilden, Germany) and TRIzol reagent (Invitrogen, Carlsbad, CA, USA) including a DNase digestion step, in accordance with the manufacturer's instructions. RNA yield and purity was assessed by determining the absorbance at 230 , 260 and $280 \mathrm{~nm}$ in a ND-1000 spectrophotometer (NanoDrop, Wilmington, DE, USA).

Illumina deep-sequencing and analysis of miRNAs. Small RNA libraries were generated as follows: i) 3'-RNA adapters were ligated to total RNA; ii) 5'-RNA adapters were ligated to total RNA; iii) ligation products were reverse transcribed to cDNA and amplified by PCR; iv) 130-155 bp PCR amplified products (correspond to small RNA fragments ranging from 15-35 nucleotides) were isolated and purified from polyacrylamide gels. After quantified with 2100 Bioanalyzer (Agilent, Palo Alto, CA, USA), the entire libraries were denatured with $0.1 \mathrm{M} \mathrm{NaOH}$ and subsequently loaded on the cBot instrument for amplification in situ using TruSeq Rapid SR cluster kit (both from Illumina, San Diego, CA, USA), as recommended by the manufacturer. Finally, small RNA libraries were quantified and sequenced for 36 cycles on Illumina HiSeq 2000 analyzer using TruSeq Rapid SBS kit (Illumina) following the manufacturer's standard workflow.

After sequencing was complete, image analysis and base calling were performed by utilizing Off-Line Basecaller software (version 1.8.0; Illumina). Clean reads which passed Solexa Chastity quality filter, were trimmed to remove the 3'-adapter sequence, and the reads shorter than 15 nucleotides were discarded. To identify known miRNAs, trimmed reads were compared with the human miRNA precursors in miRBase database (version 19.0, http://www.mirbase.org/) by using Novoalign software (release v2.07.11, http://www. novocraft.com/). Subsequently, raw counts of miRNA reads were normalized to transcripts per million (TPM) values. Differentially expressed miRNAs between samples may be 
identified by the fold-change filtering using a threshold of at least 2-fold-changes according to the normalized most abundant tag counts.

Transcription factor array and data analysis. Transcription factor array analysis was performed using TranSignal Combo Protein/DNA arrays (Panomics, Redwood City, CA, USA) according to the manufacturer's directions, which enables simultaneous detection of 345 major transcription factors. In general, nuclear extracts were prepared using the nuclear extract kit (Panomics) and the protein concentrations were measured with BCA protein assay kit (KangChen, Shanghai, China) as per the manufacturer's instructions. Ten micrograms of nuclear proteins were incubated for $30 \mathrm{~min}$ at $15^{\circ} \mathrm{C}$ with $10 \mu \mathrm{l}$ of probe mixture containing 345 biotin-labeled TF-specific DNA oligonucleotides (Panomics) to allow the formation of TF/DNA complexes. Such complexes were separated from the free probes using spin column separation system (Panomics). The TF-specific probes were then extracted from the TF/DNA complexes and hybridized to the TranSignal array membrane at $42^{\circ} \mathrm{C}$ overnight. After three washings and addition of horseradish peroxidase (HRP)-conjugated streptavidin, hybridization signals were generated by standard chemiluminescence procedures with ECL-Hyperfilm (Amersham Pharmacia Biotech, Uppsala, Sweden). The signals were detected with a GBox Imaging system (Syngene, Cambridge, UK) and spot intensities were analyzed using ScanAlyze software (version 1.0.3, http://graphics.stanford. edu/software/scanalyze/). The data points showing at least 2-fold signal difference between B-ALL samples and their controls are considered significant.

Quantitative PCR of miRNA and TF. Total RNA samples were reverse transcribed (RT) to generate cDNA with Stem-loop RT primer (for miRNAs) and oligo(dT) primer (for TFs) using the SuperScript III Reverse Transcriptase kit (Invitrogen) following the instructions of the manufacturer. QPCR was carried out in triplicate on a Prism 7500 Real-time PCR system (Applied Biosystems, Foster City, CA, USA). The reaction mixtures consisted of $5 \mu \mathrm{l}$ of $2 \mathrm{X}$ SYBR-Green PCR master mix (Applied Biosystems), $2 \mu \mathrm{l}$ of template cDNA, $0.5 \mu \mathrm{l}$ of $10 \mu \mathrm{M}$ PCR forward primer, $0.5 \mu \mathrm{l}$ of $10 \mu \mathrm{M}$ PCR reverse primer and $2 \mu \mathrm{l}$ of double-distilled water. The PCR reaction was conducted at $95^{\circ} \mathrm{C}$ for $10 \mathrm{~min}$, followed by 40 cycles of $95^{\circ} \mathrm{C}$ for $10 \mathrm{sec}$ and $60^{\circ} \mathrm{C}$ for $60 \mathrm{sec}$. To evaluate the reaction specificity, a melting curve analysis was performed immediately after PCR amplification. The expression levels of miRNAs and IFs were normalized to those of the human U6 snRNA and $18 \mathrm{~S}$ rRNA, respectively. Relative expression was analyzed using the comparative $2^{-\Delta \Delta \mathrm{Ct}}$ method (27). Differences between the two groups were compared using the Student's t-test and considered significant at $\mathrm{P}<0.05$. All primers used in this study are listed in Tables I-III.

\section{Association analysis of miRNA and TF expression profiles}

Identification of miRNA-gene/TF regulatory relationships. To acquire regulatory relationships of miRNA-gene and miRNA-TF, target prediction for differentially expressed miRNAs (fold-change, $\geq 2.0$ ) was carried out using three different online databases, including PicTar (release 2007, http://
pictar.mdc-berlin.de/cgi-bin/PicTar_vertebrate.cgi), TargetScan (release 6.2, http://www.targetscan.org/) and miRanda (version v5,http://www.ebi.ac.uk/enright-srv/microcosm/htdocs/ targets/v5/) $(28,29)$. To reduce false-positive results, only the targets predicted by at least two of the aforementioned databases were retained in this study $(28,29)$. The identified targets were then incorporated into the experimentally verified targets which were obtained from miRTarBase (release 4.5, http://mirtarbase. mbc.nctu.edu.tw/) (30). After compiling B-ALL candidate genes/TFs from the MalaCards database (version 1.08.564, http://www.malacards.org/) (31), these results were overlapped with B-ALL candidate genes/TFs to avoid the redundancy and form miRNA $\rightarrow$ gene and miRNA $\rightarrow$ TF regulatory relationships. The extracted miRNA and target gene/TF pairs were subsequently subjected to TFBS analysis.

Identification of TF-gene/miRNA regulatory relationships. TF-gene and TF-miRNA regulatory relations were analyzed using the predicted TFBS information obtained from the TFBS Conserved Track (http://genome.ucsc.edu/cgi-bin/ hgTables?hgsid=350051003\&hgta_doSchemaDb=hg19\&hgta_ doSchemaTable $=$ tfbsConsFactors) at UCSC Genome Browser database (32), and TFBSs were required to be conserved among human/mouse/rat. To reduce false-positive predictions, a P-value of 0.05 was selected as a cut-off. Next, the 5,000 bp upstream to $1,000 \mathrm{bp}$ downstream of the transcription start site (TSS) was defined as putative promoter region for each gene $(33,34)$. A gene is considered to be the target of a TF when at least one TFBS is located within the gene's promoter region and its $\mathrm{P}<0.05$. As a part of the common transcription unit, miRNAs usually co-expressed with known host genes (protein coding genes/lncRNAs). Therefore, the putative promoter region (spans from 5,000 bp upstream to $1,000 \mathrm{bp}$ downstream of the TSS) for host genes is used as the miRNA promoter $(34,35)$. miRNAs having a TFBS that completely falls within the promoter region of their host gene and its $\mathrm{P}<0.05$ were considered as TF targets. To further increase the prediction accuracy, the predicted TFs were then overlapped with differentially expressed TFs (fold-change, $\geq 2.0$ ) obtained from the TF profiling data. After extraction of the TF-miRNA pairs and TF-miRNA target gene pairs, the predicted $\mathrm{TF} \rightarrow \mathrm{miRNA}$ and $\mathrm{TF} \rightarrow$ gene relations were retrieved, respectively.

miRNA-TF regulatory network generation and network node analysis. By merging the regulatory relationships between TFs, miRNAs and target genes $(\mathrm{TF} \rightarrow \mathrm{miRNA}, \mathrm{TF} \rightarrow$ gene, miRNA $\rightarrow$ gene and miRNA $\rightarrow \mathrm{TF}$ ), we constructed a comprehensive miRNA-TF regulatory network for B-ALL using Gephi software (release 0.8.1- $\beta$, http://gephi.github.io/). GO database (www.geneontology. org) (36) and Kyoto Encydopedia of Gene and Genomes (KEGG) database (http://www.genome.jp/kegg/) (37) were then used to analyze the functional categories and pathways of the network nodes, which were considered to be significant when $\mathrm{P}<0.05$. For further evaluation of network characteristics, the node degree is determined by calculating the number of direct links between nodes within the network. To identify network hubs, we classified the nodes by descending based on their degrees. The nodes having a total degree (sum of the in-degree and out-degree) $\geq 10$ were considered as the hub components. Any neighboring node connected to hubs was extracted from the network to construct the sub-network. 
Table I. Primers used for cDNA synthesis of miRNAs.

\begin{tabular}{ll}
\hline miRNAs & \multicolumn{1}{c}{ Primer for cDNA synthesis $\left(5^{\prime}-3^{\prime}\right)$} \\
\hline miR-200c-3p & CTCAACTGGTGTCGTGGAGTCGGCAATTCAGTTGAGTCCAT \\
miR-200b-3p & CTCAACTGGTGTCGTGGAGTCGGCAATTCAGTTGAGTCATCATT \\
miR-194-5p & CTCAACTGGTGTCGTGGAGTCGGCAATTCAGTTGAGTCCACATG \\
miR-26b-5p & GTCGTATCCAGTGCGTGTCGTGGAGTCGGCAATTGCACTGGATACGACACCTAT \\
miR-16-5p & GTCGTATCCAGTGCGTGTCGTGGAGTCGGCAATTGCACTGGATACGACCGCCAAT \\
miR-424-5p & GTCGTATCCAGTGCGTGTCGTGGAGTCGGCAATTGCACTGGATACGACTTCAAA \\
U6 snRNA & CGCTTCACGAATTTGCGTGTCAT
\end{tabular}

Table II. Primers used for quantitative PCR of miRNAs.

\begin{tabular}{lllc}
\hline miRNAs & \multicolumn{1}{c}{ Sense primer (5'-3') } & \multicolumn{1}{c}{ Antisense primer (5'-3') } & Product (bp) \\
\hline miR-200c-3p & ACACTCCAGCTGGGTAATACTGCCGGGTAATG & CTCAACTGGTGTCGTGGA & 73 \\
miR-200b-3p & ACACTCCAGCTGGGTAATACTGCCTGGTAATG & CTCAACTGGTGTCGTGGA & 73 \\
miR-194-5p & ACACTCCAGCTGGGTGTAACAGCAACTCCATG & CTCAACTGGTGTCGTGGA & 73 \\
miR-26b-5p & GGGGTTCAAGTAATTCAGG & TGCGTGTCGTGGAGTC & 65 \\
miR-16-5p & GGGTAGCAGCACGTAAATA & CAGTGCGTGTCGTGGAGT & 65 \\
miR-424-5p & GGGCAGCAGCAATTCATGT & GTGCGTGTCGTGGAGTCG & 63 \\
U6 snRNA & GCTTCGGCAGCACATATACTAAAAT & CGCTTCACGAATTTGCGTGTCAT & 89 \\
\hline
\end{tabular}

Table III. Primers used for quantitative PCR of TFs.

\begin{tabular}{lllr}
\hline TFs & \multicolumn{1}{c}{ Sense primer $\left(5^{\prime}-3^{\prime}\right)$} & \multicolumn{1}{c}{ Antisense primer (5'-3') } & Product (bp) \\
\hline STAT5A & GGCCATCCTAGGTTTTGTG & CCCGATTTCTGAGTCACTAAAG & 100 \\
FOXD1 & CTTTTCTCGTCTTGGTGGTT & CAGTTTTGTCCGAGAATTCG & 104 \\
MEF2A & AGTTTCCAACAGCTGTCCTT & GATTGGGACCACCTGTGTAC & 90 \\
FOXF2 & CACTCCAGCATGTCCTCCTA & CTAGCTGAGGGATGGAAAGA & 170 \\
HLF & CGATCACCTTCTGCTCCTA & GATTCGGATAAGGCTTTGC & 110 \\
FOXO4 & ATAGCACCACCTCCAGTCA & CATGTCACACTCCAGGTTCTC & 150 \\
18S rRNA & CCTGGATACCGCAGCTAGGA & GCGGCGCAATACGAATGCCCC & 112 \\
\hline
\end{tabular}

\section{Results}

Alterations in miRNA and TF expression in adult B-ALL. To acquire B-ALL-related miRNAs and TFs, we analyzed the global expression change of miRNAs and TFs in adult B-ALL samples compared with their controls, using Illumina deep-sequencing and TF array technology, respectively. The miRNA expression profiling data showed 291 miRNAs were differentially expressed (fold-change, $\geq 2.0$ ) among groups (Fig. 1A), including 168 that were upregulated and 123 that were downregulated. miR-1246 (fold-change, 6516.2) was the most upregulated miRNA, while miR-106b-5p (fold-change, 1509.5) was the most downregulated miRNA.

Analysis of the TF array results revealed 201 TFs (57 upregulated and 144 downregulated) that were differentially expressed (fold-change $\geq 2.0$ ) between the control and adult B-ALL samples (Fig. 1B). Among these TFs, MYC (fold-change, 36.0) exhibited the highest degree of upregulation, while MYOG (fold-change, 91.4) was the most downregulated TF.

Verification of Illumina sequencing and TF array results by $q P C R$. To demonstrate the reliability of the Illumina sequencing and TF array data, 6 miRNAs and 6 TFs that are differentially expressed were chosen for further verification by qPCR. As shown in Fig. 2A, miR-200c-3p, miR-200b-3p and miR-194-5p were upregulated and that miR-26b-5p, miR-16-5p and miR-424-5p were downregulated in adult B-ALL samples relative to their controls (all $\mathrm{P}<0.05$ ). Furthermore, qPCR analysis also confirmed statistically significant differences between groups in signal transducer and activator of transcription 5A (STAT5A), FOXD1, MEF2A, FOXF2, HLF and 
A

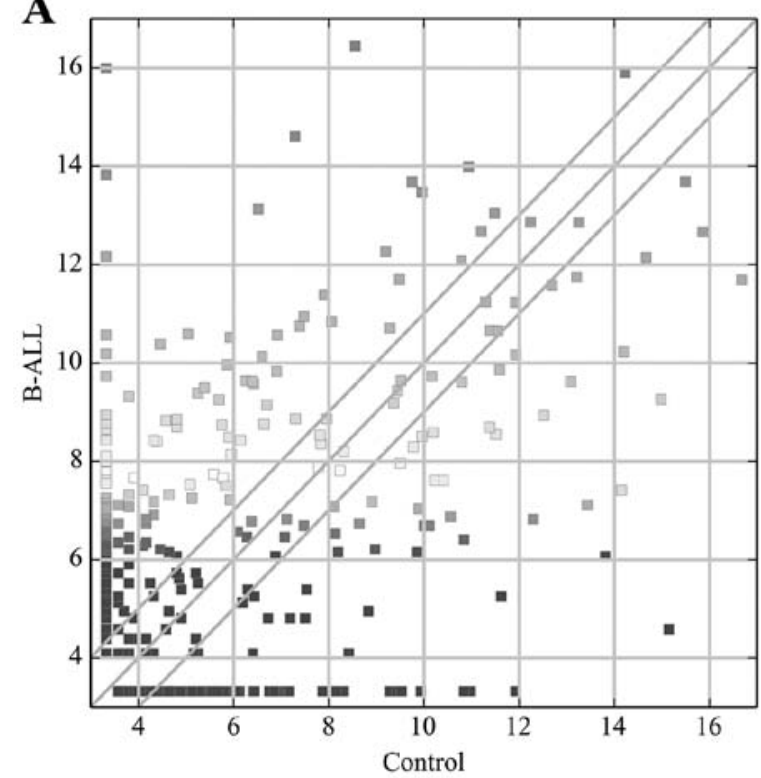

B

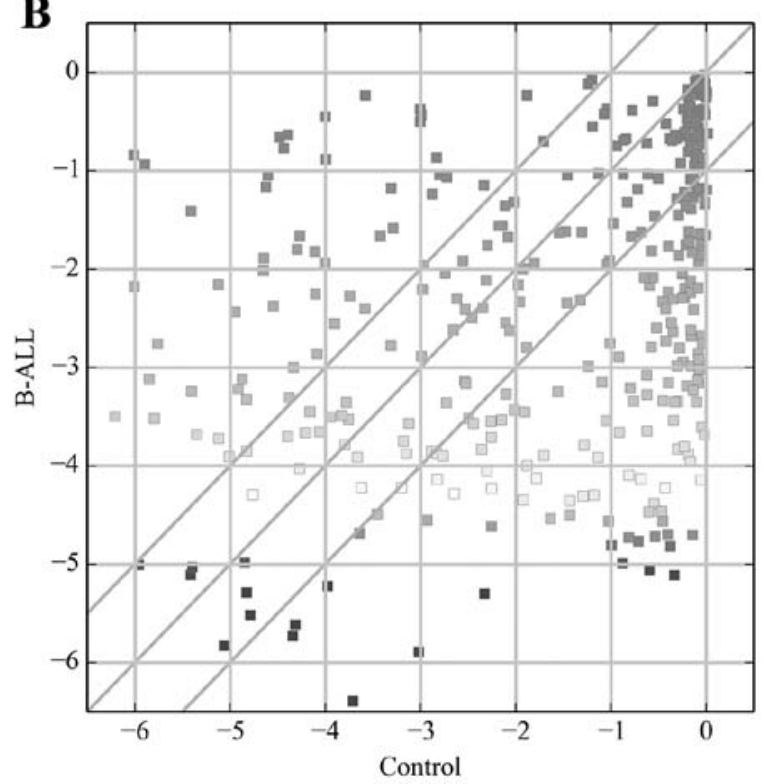

Figure 1. miRNA and TF profile contrasting between the bone marrow samples of B-ALL patients and control groups. The scatter-plot is used for assessing the miRNA (A) and TF (B) expression change in B-ALL samples relative to their control. The values of x-axis and y-axis in the scatter-plot represent averaged normalized values for each group ( $\log _{2}$ scaled). The oblique lines are fold-change lines (fold-change cutoff value was set to 2.0 ).
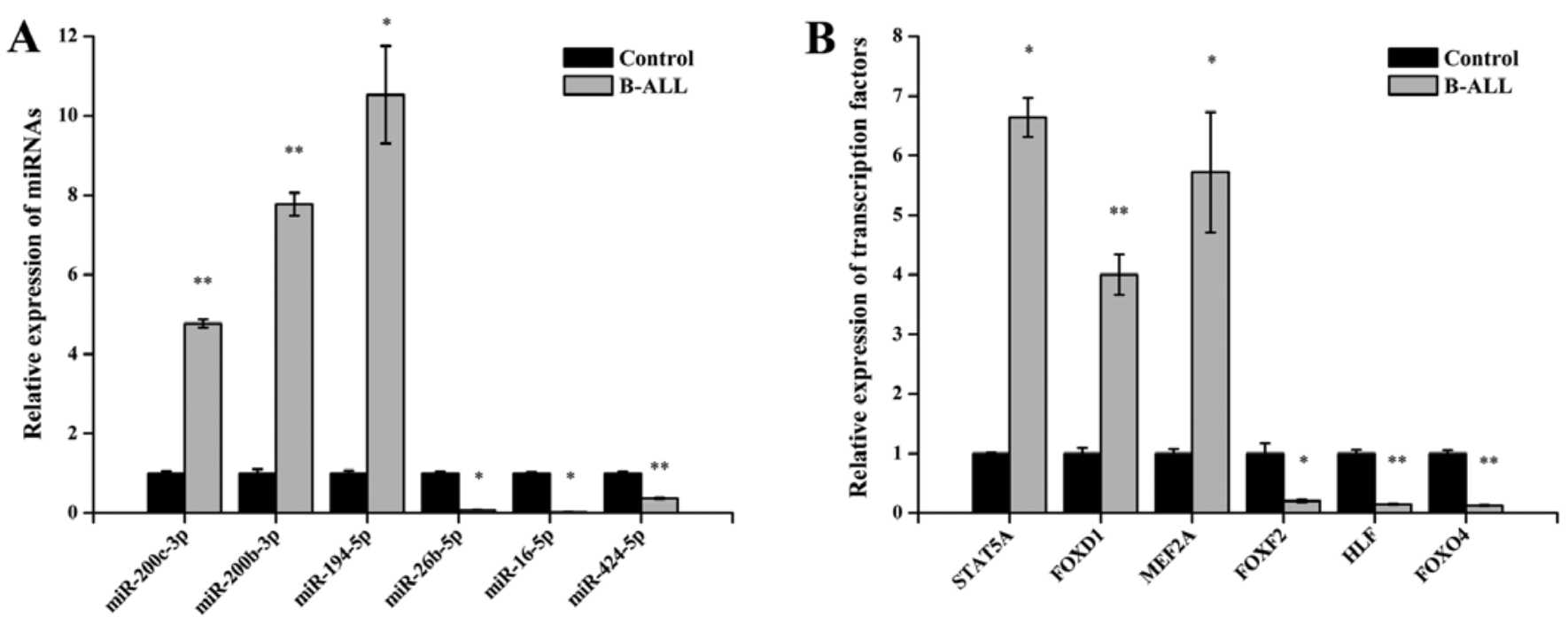

Figure 2. Validation of Illumina sequencing and TF array data by qPCR. The relative expression level of miRNAs (A) and IFs (B) were normalized to those of the human U6 snRNA and 18S rRNA, respectively. Data displayed in histograms are means $\pm \mathrm{SD},{ }^{*} \mathrm{P}<0.05$ and ${ }^{* * *} \mathrm{P}<0.01$ comparing $\mathrm{B}-\mathrm{ALL}$ samples with control samples (Student's t-test).

FOXO4 (P<0.05 for each TFs) (Fig. 2B). These results were consistent with the Illumina sequencing and $\mathrm{TF}$ array data.

miRNA and TF regulatory network in adult B-ALL. After merging the four types of regulatory interactions (miRNA $\rightarrow$ gene, miRNA $\rightarrow \mathrm{TF}, \mathrm{TF} \rightarrow$ gene and $\mathrm{TF} \rightarrow \mathrm{miRNA}$ ) identified in the Materials and methods section, we constructed a miRNA-TF regulatory network specific for adult B-ALL (Fig. 3). A total of 41 miRNA-regulated TFs and 19 TFs regulated miRNAs in the network. On average, each miRNA was regulated by 0.24 TFs, whereas each TF was regulated by 10.25 miRNAs. The numbers of nodes and regulatory relationships of the resultant network are listed in Table IV. FFLs and FBLs are motifs known to play key roles in gene regulatory networks (21). Typically, FFLs can be categorized into three different types based on the master regulators: miRNA-FFL, TF-FFL and composite FFL $(21,38)$. In Table $\mathrm{V}$, we summarized the FFLs and FBLs in the B-ALL network and found $\sim 98 \%$ of motifs were FFL. Among the 526 merged FFLs, 492 (93.5\%) were TF-FFLs, in which TF was the master regulator, $30(5.7 \%)$ belonged to miRNA-FFLs, and $4(0.8 \%)$ belonged to composite-FFLs. These results indicate that TF-FFL was the dominant motif in the network.

Functional analysis of the miRNA and TF mediated regulatory network. Enrichment analysis is an effective method to understand functional nodes and network motifs. To investigate the 


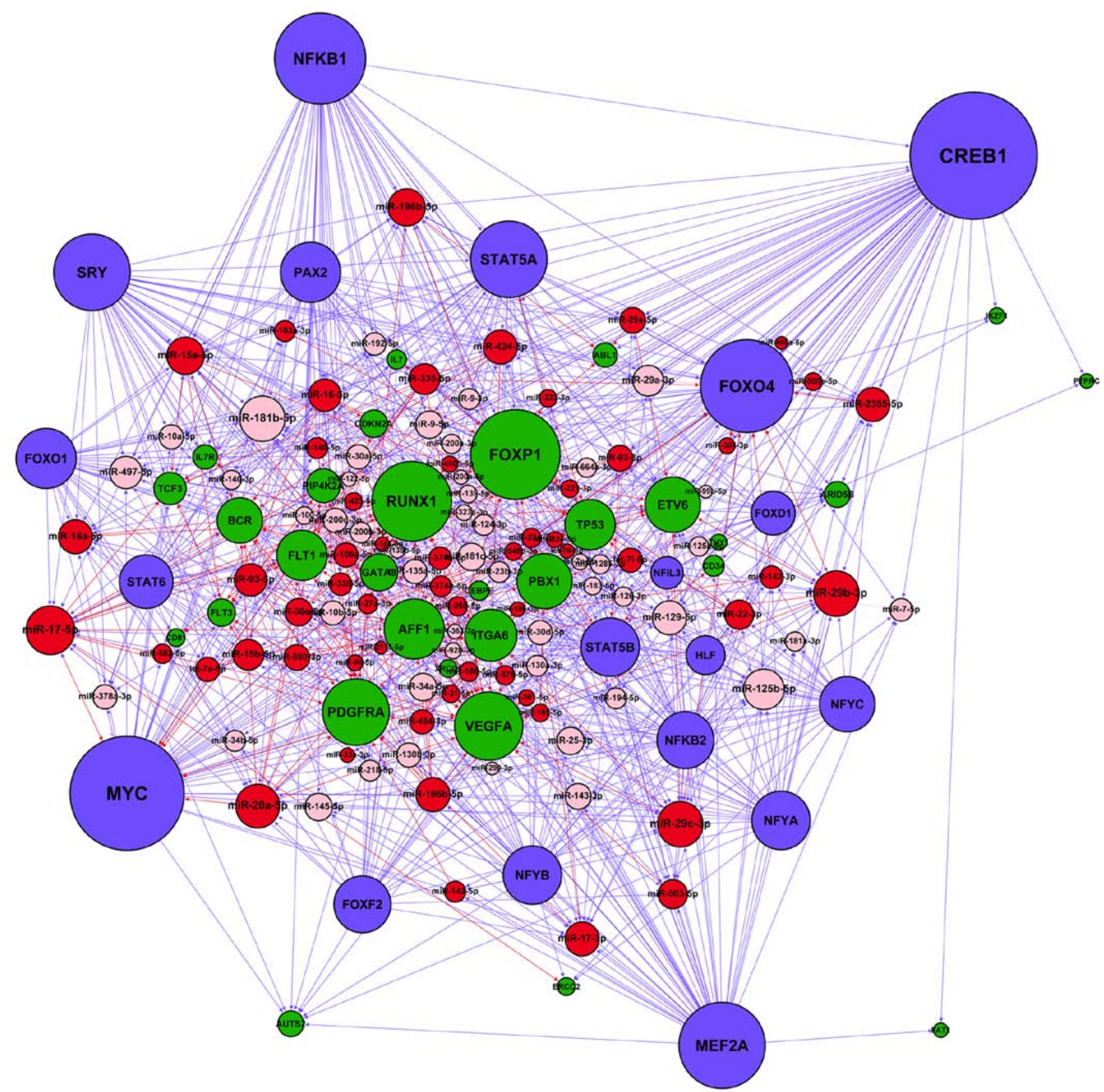

Figure 3. miRNA-TF regulatory network in B-ALL. Pink nodes, upregulated B-ALL-related miRNAs; red nodes, downregulated B-ALL-related miRNAs; green nodes, B-ALL candidate genes; blue nodes, TFs. Pink arrows, regulatory relationships for upregulated miRNA and target gene; red arrows, regulatory relationships for downregulated miRNA and target gene; blue arrows, regulatory relationships for TF-miRNA or TF-gene.

functional categories of the B-ALL miRNA-TF regulatory network, we performed the GO enrichment analysis for all network nodes and identified 10 highly-enriched biological process terms (Fig. 4). Among these GO terms, 5 terms were related to the initiation and progression of B-ALL, such as positive regulation of gene expression, hemopoietic or lymphoid organ development, hemopoiesis, positive regulation of transcription (DNA-dependent) and positive regulation of macromolecule biosynthetic process $(6,7,11,39)$. Moreover, significantly-enriched biological pathways for the network nodes were also examined by applying KEGG pathway enrichment analysis. The nodes were significantly enriched in 25 different pathways, of which 13 pathways were directly related to leukemia or hematopoiesis (Table VI). Among these pathways, PI3K-Akt signaling pathway, Jak-STAT signaling pathway, Ras signaling pathway and cell cycle pathway were well-known to be involved in B-ALL $(6,9,11,13,39)$.

Network topological analysis and sub-network construction. To assess the global properties of this network, we calculated degrees and analyzed degree distributions for all nodes, which are primary topological network measures. In the network, the average node degree of miRNAs, TFs and genes was 7.6 (range from 1 to 23), 35 (range from 13 to 71) and 15.7 (range from 


\section{*Positive regulation of gene expression \\ Positive regulation of macromolecule metabolic process \\ *Hemopoietic or lymphoid organ development \\ Positive regulation of metabolic process \\ Immune system development}

Positive regulation of transcription from RNA polymerase II promoter

${ }^{\text {*} H e m o p o i e s i s ~}$

*Positive regulation of transcription (DNA-dependent)

${ }^{*}$ Positive regulation of macromolecule biosynthetic process

Regulation of transcription from RNA polymerase II promoter

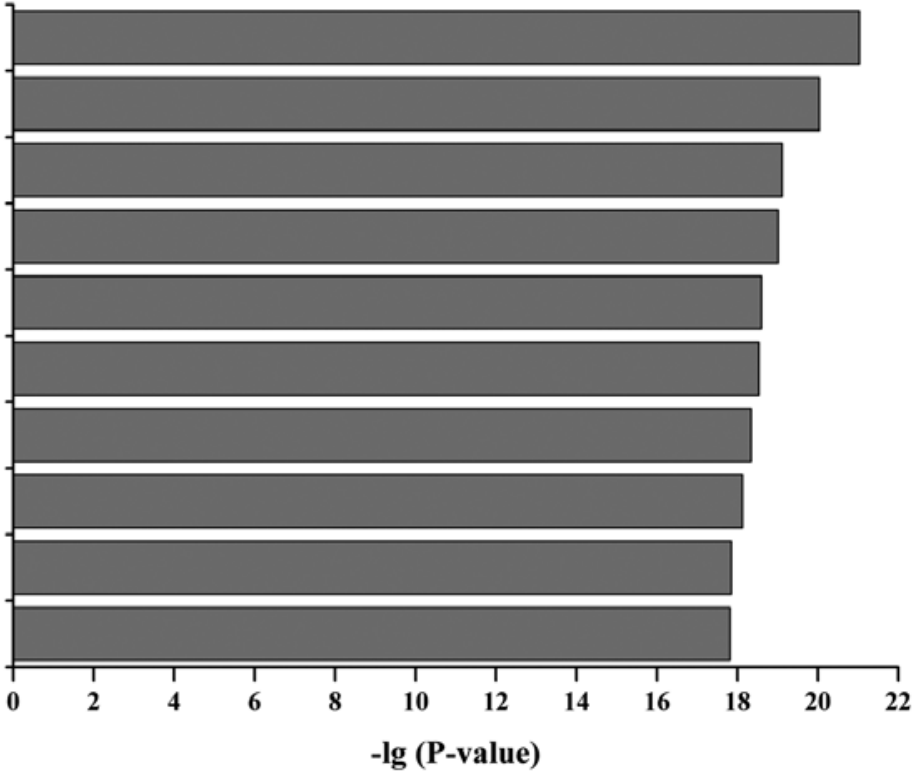

Figure 4. Highly-enriched biological process terms for nodes in the B-ALL miRNA-TF regulatory network. Gene ontology (GO) analysis was carried out to examine the enrichment of network nodes in biological processes. $\mathrm{P}<0.05$ was considered to be significant, and the asterisk (*) indicates the terms related to B-ALL in GO.

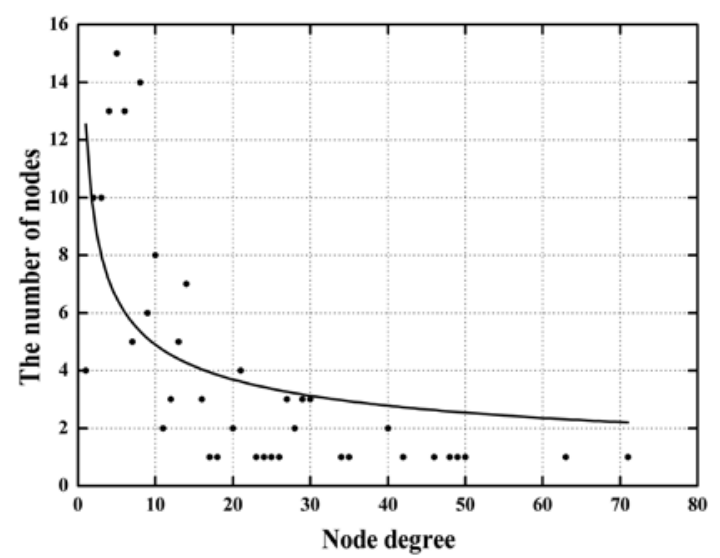

Figure 5. Degree distribution of all nodes in the B-ALL miRNA-TF regulatory network. The $\mathrm{x}$-axis represents the degree of the nodes, and the $\mathrm{y}$-axis represents the number of nodes for each degree.
Table IV. Summary of relationships in the B-ALL-related miRNA and transcription factor (TF) regulatory network.

\begin{tabular}{lcccc}
\hline Relationship & $\begin{array}{c}\text { No. of } \\
\text { pairs }\end{array}$ & $\begin{array}{c}\text { No. of } \\
\text { miRNAs }\end{array}$ & $\begin{array}{c}\text { No. of } \\
\text { genes }\end{array}$ & $\begin{array}{c}\text { No. of } \\
\text { TFs }\end{array}$ \\
\hline miRNA-gene $^{\mathrm{a}}$ & 431 & 131 & 27 & - \\
miRNA-TF $^{\mathrm{b}}$ & 47 & 41 & - & 4 \\
TF-gene $^{\mathrm{c}}$ & 176 & - & 35 & 19 \\
TF-miRNA $^{\mathrm{d}}$ & 429 & 80 & - & 19 \\
\hline
\end{tabular}

${ }^{\mathrm{a}}$ miRNA repression of gene expression; ${ }^{\mathrm{b}}$ miRNA repression of $\mathrm{TF}$ expression; ${ }^{\mathrm{C}} \mathrm{TF}$ regulation of gene expression; ${ }^{\mathrm{d}} \mathrm{TF}$ regulation of miRNA expression.

Table V. Summary of feed-forward loops (FFLs) and feedback loops (FBLs) based on B-ALL-related network data.

\begin{tabular}{|c|c|c|c|c|c|c|c|c|}
\hline \multirow[b]{2}{*}{ Motif types } & \multirow[b]{2}{*}{ No. of motifs } & \multicolumn{3}{|c|}{ No. of nodes } & \multicolumn{4}{|c|}{ No. of links } \\
\hline & & Genes & miRNAs & TFs & miRNA-gene & miRNA-TF & TF-gene & TF-miRNA \\
\hline miRNA-FFL & 30 & 9 & 15 & 4 & 27 & 19 & 16 & - \\
\hline TF-FFL & 492 & 20 & 70 & 19 & 189 & - & 104 & 280 \\
\hline Composite-FFL & 4 & 4 & 2 & 2 & 4 & 2 & 4 & 2 \\
\hline FBL & 9 & - & 9 & 3 & - & 9 & - & 9 \\
\hline
\end{tabular}

2 to 48), respectively. These results indicate that TFs are located at higher degrees within the network. Only a few nodes displayed a high node degree (hubs) while most nodes had a low degree, which reflected a scale-free feature of this network and further demonstrate that hub nodes may play vital roles in the network stability and robustness (Fig. 5). We categorized the nodes in descending order according to their total degrees and identified hub nodes (total degrees $\geq 10$ ) inside 


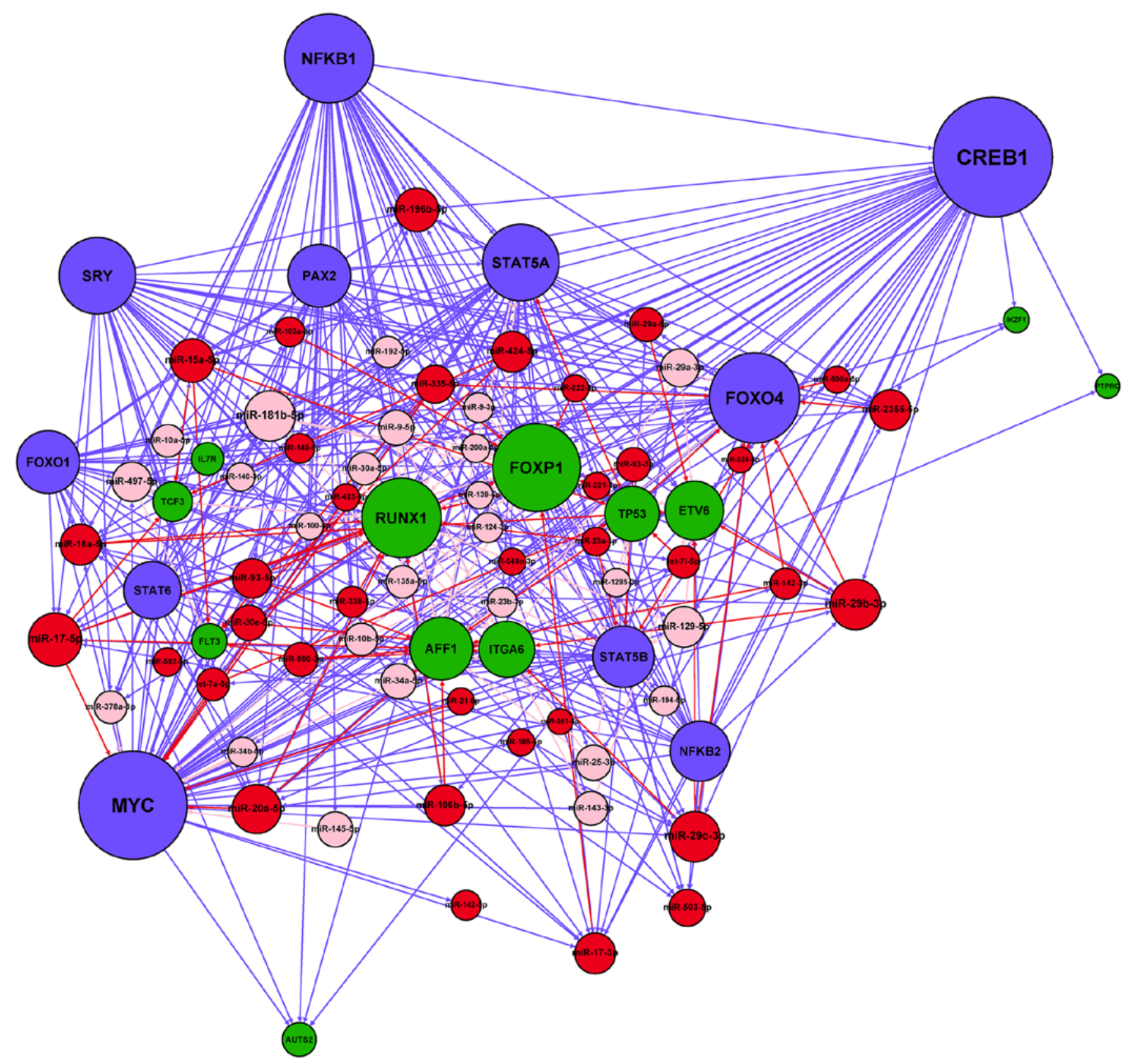

Figure 6. B-ALL-related Jak-STAT signaling sub-network. The symbols of different nodes and arrows are the same as Fig. 3.

the network. Twenty-eight hub miRNAs and 19 hub TFs were obtained from the network, respectively (Tables VII and VIII). Notably, 6 of the 28 hub miRNAs belonged to members of the miR-15 family, namely miR-15a-5p/15b-5p/16-5p/424-5p/497$5 \mathrm{p} / 503-5 \mathrm{p}$ (Table VII), indicating an important role of miR-15 family in B-ALL-related regulatory network. Moreover, more than half of the 19 hub TFs were either well-known leukemia regulators, such as MYC $(40)$, STAT5A $(41,42)$ and STAT5B $(41,42)$, or associated with leukemia development and progression, such as CREB1 (43), FOXO1 (44), FOXO4 (45), NFKB1 (46), NFKB2 (47), STAT6 (48) and HLF $(11,49)$, which preliminarily reflected the robustness of our network. Among the 47 hub regulators, 4 hub miRNAs [miR-17-5p (19), miR-125b-5p (50), miR-18a-5p (19) and miR-34a-5p (4)] and 5 hub TFs [MYC $(43,51,52)$, STAT5A $(41,53)$, STAT5B $(41,54)$, FOXO1 (44) and HLF (11)] have been previously implicated in B-ALL. Of these regulators, 3 hub TFs (MYC, STAT5A and STAT5B) participated in Jak-STAT signaling pathway which was directly related to B-ALL pathogenesis. Therefore, we constructed a sub-network from these 3 TFs and all their direct linked nodes in the network, and defined it as the B-ALL-related Jak-STAT signaling sub-network, which included 436 edges, 12 genes, 61 miRNAs and 11 TFs (Fig. 6).

\section{Discussion}

B-ALL is a genetically heterogeneous disease and many different types of cytogenetic abnormalities and molecular genetic mutations are important initiating events in the leukemogenesis of B-ALL $(6,11,39)$. However, these genetic alterations are insufficient to fully explain the biology and heterogeneity of this disease (11). The pathogenesis of B-ALL 
Table VI. Pathway analysis for nodes in B-ALL-related miRNA and transcription factor regulatory network.

\begin{tabular}{|c|c|c|c|c|}
\hline Pathway ID & Definition & Gene count & $\%$ & Fisher-P-value \\
\hline hsa05200 & Pathways in cancer ${ }^{\mathrm{a}}$ & 15 & 9.43 & 7.67E-11 \\
\hline hsa05202 & Transcriptional misregulation in cancer ${ }^{\mathrm{a}}$ & 12 & 7.55 & $1.23 \mathrm{E}-10$ \\
\hline hsa05220 & Chronic myeloid leukemia $^{a}$ & 9 & 5.66 & $1.54 \mathrm{E}-10$ \\
\hline hsa05166 & HTLV-I infection ${ }^{\mathrm{a}}$ & 11 & 6.92 & $1.10 \mathrm{E}-07$ \\
\hline hsa05221 & Acute myeloid leukemia ${ }^{a}$ & 6 & 3.77 & $6.18 \mathrm{E}-07$ \\
\hline hsa05161 & Hepatitis B & 7 & 4.40 & $1.37 \mathrm{E}-05$ \\
\hline hsa04151 & PI3K-Akt signaling pathway ${ }^{\mathrm{a}}$ & 10 & 6.29 & $1.42 \mathrm{E}-05$ \\
\hline hsa05219 & Bladder cancer & 4 & 2.52 & $5.48 \mathrm{E}-05$ \\
\hline hsa04640 & Hematopoietic cell lineage $^{a}$ & 5 & 3.14 & 0.000120 \\
\hline hsa05203 & Viral carcinogenesis ${ }^{\mathrm{a}}$ & 7 & 4.40 & 0.000125 \\
\hline hsa05215 & Prostate cancer & 5 & 3.14 & 0.000126 \\
\hline hsa05206 & MicroRNAs in cancer & 8 & 5.03 & 0.000191 \\
\hline hsa04630 & Jak-STAT signaling pathway ${ }^{\mathrm{a}}$ & 6 & 3.77 & 0.000206 \\
\hline hsa05212 & Pancreatic cancer & 4 & 2.52 & 0.000478 \\
\hline hsa04612 & Antigen processing and presentation & 4 & 2.52 & 0.000945 \\
\hline hsa05222 & Small cell lung cancer & 4 & 2.52 & 0.001299 \\
\hline hsa04012 & ErbB signaling pathway & 4 & 2.52 & 0.001356 \\
\hline hsa04014 & Ras signaling pathway ${ }^{\mathrm{a}}$ & 6 & 3.77 & 0.001509 \\
\hline hsa05152 & Tuberculosis & 5 & 3.14 & 0.003049 \\
\hline hsa04060 & Cytokine-cytokine receptor interaction & 6 & 3.77 & 0.003687 \\
\hline hsa04110 & Cell cycle ${ }^{a}$ & 4 & 2.52 & 0.004927 \\
\hline hsa05162 & Measles & 4 & 2.52 & 0.006479 \\
\hline hsa04010 & MAPK signaling pathwaya & 5 & 3.14 & 0.013771 \\
\hline hsa05169 & Epstein-Barr virus infection ${ }^{\mathrm{a}}$ & 4 & 2.52 & 0.026308 \\
\hline hsa04510 & Focal adhesion & 4 & 2.52 & 0.027579 \\
\hline
\end{tabular}

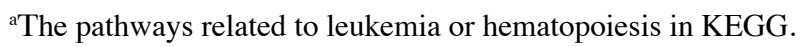

is still not entirely clear to date. In particular, the heterogeneity and complexity makes it difficult to discover hub regulators and regulatory motifs involved in the pathogenesis of B-ALL. By integrating high-throughput expression profiling technology and bioinformatics approaches, systems biology focuses on exploring the interactions between biological molecules and explaining the biological phenomenon on the network level, which may accelerate the discovery of hub regulators and pathways that control various aspects of the disease pathogenesis $(55,56)$.

To improve our understanding of the molecular mechanism of B-ALL, we applied systems biology approach to obtain and integrate expression changes of miRNAs and TFs between bone marrow samples from adult B-ALL patients and their controls, and constructed a miRNA-TF mediated regulatory network associated with this disease (Fig. 3). Subsequently, we identified the miRNA and TF mediated regulatory motifs from the network, including FFLs and FBLs (Table V), which are significant and prevalent motifs in gene regulatory networks (21). It has been reported that FFLs may work as the core of the global gene regulatory network and play critical roles in disease pathogenesis $(23,57)$. The majority of FFLs in our network are TF-FFLs, in which $\mathrm{TF}$ as the main regulator controls the expression of both miRNA and their common target gene. This finding is consistent with a recent study that TF-FFL was the dominant motif in the development of B lymphocytes (57), revealing an important role of TF-FFL in B cell development and B-ALL pathogenesis.

For evaluating the functional properties of this network, GO and KEGG pathway annotation were used to analyze the enrichment of the network nodes (Fig. 4 and Table VI). Among 10 highly-enriched biological process terms, one half of these terms were related to the occurrence and development of B-ALL. Moreover, more than half of significantly-enriched pathways were directly associated with leukemia or hematopoiesis. Among them, four pathways have been reported to be implicated in B-ALL pathogenesis, such as PI3K-Akt signaling pathway, Jak-STAT signaling pathway, Ras signaling pathway and cell cycle pathway. Further network topological analysis identified 28 hub miRNAs and 19 hub TFs within the network (Tables VII and VIII). Of these, 4 hub miRNAs (miR-17-5p, miR-125b-5p, miR-18a-5p and miR-34a-5p) and 5 hub TFs (MYC, STAT5A, STAT5B, FOXO1 and HLF) were functionally associated with B-ALL, which have been confirmed in previous studies. Collectively, these results preliminarily verified the reliability of our network. Among 
Table VII. Hub miRNAs in B-ALL-related miRNA and transcription factor regulatory network.

\begin{tabular}{clccc}
\hline & & & & Total \\
Top & miRNA & In-degree & Out-degree & degree \\
\hline 1 & miR-17-5p & 12 & 11 & 23 \\
2 & miR-181b-5p & 12 & 9 & 21 \\
3 & miR-29b-3p & 17 & 4 & 21 \\
4 & miR-29c-3p & 17 & 4 & 21 \\
5 & miR-20a-5p & 12 & 8 & 20 \\
6 & miR-125b-5p & 11 & 7 & 18 \\
7 & miR-196b-5p & 13 & 3 & 16 \\
8 & miR-15a-5p & 11 & 5 & 16 \\
9 & miR-129-5p & 8 & 6 & 14 \\
10 & miR-18a-5p & 12 & 2 & 14 \\
11 & miR-17-3p & 12 & 2 & 14 \\
12 & miR-2355-5p & 13 & 1 & 14 \\
13 & miR-424-5p & 10 & 4 & 14 \\
14 & miR-106b-5p & 6 & 8 & 14 \\
15 & miR-497-5p & 9 & 4 & 13 \\
16 & miR-93-5p & 6 & 7 & 13 \\
17 & miR-16-5p & 7 & 6 & 13 \\
18 & miR-29a-3p & 7 & 5 & 12 \\
19 & miR-15b-5p & 7 & 5 & 12 \\
20 & miR-335-5p & 5 & 7 & 12 \\
21 & miR-22-3p & 9 & 2 & 11 \\
22 & miR-503-5p & 10 & 1 & 11 \\
23 & miR-25-3p & 6 & 4 & 10 \\
24 & miR-9-5p & 4 & 6 & 10 \\
25 & miR-145-5p & 6 & 4 & 10 \\
26 & miR-34a-5p & 2 & 8 & 10 \\
27 & miR-181c-5p & 1 & 9 & 10 \\
28 & miR-30e-5p & 6 & 4 & 10 \\
\hline & & & & \\
& & & 5 \\
\end{tabular}

these hub TFs, MYC, STAT5A and STAT5B participated in Jak-STAT signaling pathway which is the well-known pathway involved in B-ALL. As a member of the helix-loop-helix/ leucine-zipper transcription factor family, MYC is a master regulator of important biological processes such as cell survival, proliferation, differentiation, metabolism and pluripotency maintenance $(58,59)$. Constitutive activation of MYC exhibited in a variety of B-ALL cell lines (60). Double transgenesis of MYC and Bcl-2 caused hyperproliferation of pre-B and B cells and promoted initiation of B-ALL in mice (51). Inhibition of MYC expression and STAT5 phosphorylation by JQ1 significantly prolonged survival in mice xenografted with primary human CRLF2-rearranged B-ALL (52). STAT5 is a cytoplasmic transcription factor that is usually transiently activated by phosphorylation in response to a wide variety of growth factors and cytokines (61). STAT5 consists of two separate genes, STAT5A and STAT5B, which collectively are key regulators of cell proliferation and survival in early B lymphopoiesis as well as plays an important role
Table VIII. Hub transcription factors in B-ALL-related miRNA and transcription factor regulatory network.

\begin{tabular}{ccccc}
\hline Top & $\begin{array}{c}\text { Transcription } \\
\text { factor }\end{array}$ & In-degree & Out-degree & $\begin{array}{c}\text { Total } \\
\text { degree }\end{array}$ \\
\hline 1 & CREB1 & 8 & 63 & 71 \\
2 & MYC & 14 & 49 & 63 \\
3 & FOXO4 & 18 & 32 & 50 \\
4 & NFKB1 & 0 & 49 & 49 \\
5 & MEF2A & 0 & 46 & 46 \\
6 & SRY & 0 & 40 & 40 \\
7 & STAT5A & 8 & 32 & 40 \\
8 & PAX2 & 0 & 30 & 30 \\
9 & FOXO1 & 0 & 30 & 30 \\
10 & NFYB & 0 & 29 & 29 \\
11 & NFYA & 0 & 29 & 29 \\
12 & STAT5B & 12 & 17 & 29 \\
13 & NFKB2 & 0 & 28 & 28 \\
14 & FOXF2 & 0 & 28 & 28 \\
15 & NFYC & 0 & 27 & 27 \\
16 & STAT6 & 0 & 26 & 26 \\
17 & FOXD1 & 0 & 20 & 20 \\
18 & HLF & 0 & 17 & 17 \\
19 & NFIL3 & 0 & 13 & 13 \\
\hline
\end{tabular}

in ordering immunoglobulin recombination during B cell development (41). Constitutively high expression of STAT5A induced by retrovirus integration in mice perturbed signaling in early pre-B cells leading to spontaneous pre-B-ALL/ pre-B lymphomas (62). Overexpression of STAT5A in mice cooperate with the loss of $\mathrm{p} 53$ function also promoted faster initiation of B-ALL/B-cell lymphoma (55). Furthermore, transgenic expression of STAT5B strongly synergized with adapter protein BLNK deficiency to induce pre-B-ALL in mice by inhibiting expression of p27 (54). To further investigate the regulation of MYC, STAT5A and STAT5B on highly complex molecular network in B-ALL, we subsequently constructed a B-ALL-related Jak-STAT signaling sub-network (Fig. 6) by extracting MYC, STAT5A, STAT5B and all their directly linking nodes from the network.

In the sub-network, MYC stood out as a key regulator due to its direct linking to STAT5A and STAT5B, which may establish cross-talk between MYC and all sub-network nodes. Notably, MYC and miR-15a-5p were predicted to cooperatively regulate their common target genes FMS-like tyrosine kinase 3 (FLT3), which constitute a MYC/miR-15a-5p/FLT3 TF-FFL. As a member of the miR-15 family as well as a hub miRNA in our network, miR-15a-5p is a potential tumor suppressor and is downregulated in glioma, bladder cancer, hepatoma, prostate cancer, chronic lymphocytic leukemia and different kinds of lymphoma, exerting its function by inducing apoptosis, inhibiting cell proliferation or suppressing tumorigenicity $(63,64)$. In accord with the above-mentioned reports, decreased expression of miR-15a-5p was also 


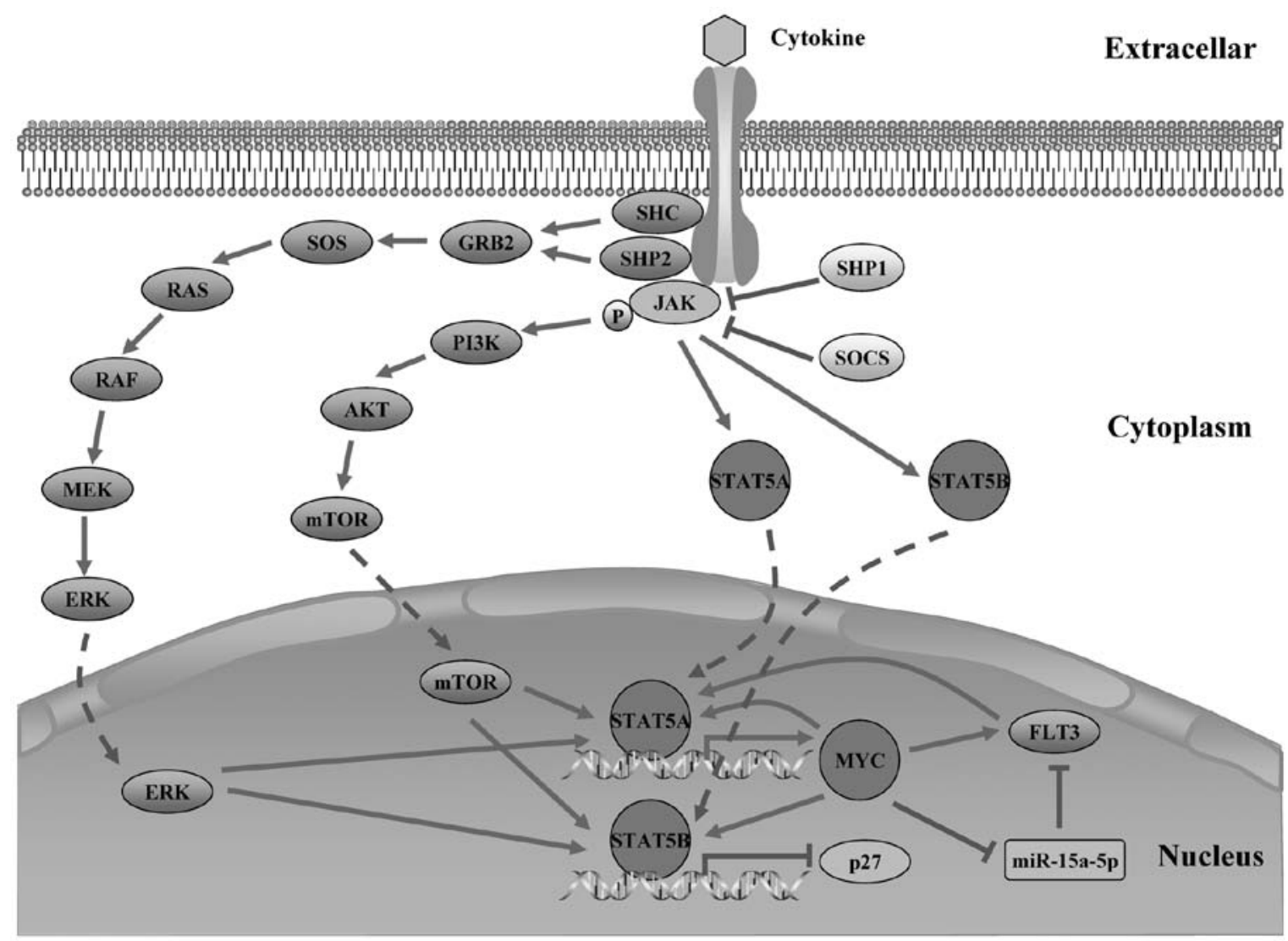

Figure 7. A schematic model of MYC/miR-15a-5p/FLT3 FFL involving in Jak-STAT signaling pathway and regulatory network in B-ALL. Round nodes, TFs; rectangle nodes, miRNAs; hexagonal nodes, cytokines; ellipse nodes, genes. Edges, sharp arrow means activation; T-shaped arrow represents repression.

observed in our study, indicating a potential relationship between miR-15a-5p and B-ALL. However, the exact effect of miR-15a-5p on B-ALL occurrence and development is still unknown. It has been reported that MYC transcriptionally repressed miR-15a-5p expression by binding to its host gene (DLEU2) promoter (58). Inhibition of miR-15a-5p expression by MYC may promote expression of their common target gene, the FLT3. Therefore, we speculate that MYC may synergize with miR-15a-5p to induce FLT3 expression in MYC/miR-15a-5p/FLT3 TF-FFL. FLT3 is a type III receptor tyrosine kinase, which is primarily expressed in lymphoid progenitors and early myeloid and plays a crucial role in their differentiation and proliferation (65). Highly expressed FLT3 was observed in nearly all pre-B-ALL and most acute myeloid leukemia (AML) patient samples as well as the majority of pre-B-ALL and AML cell lines (65-67). In B-ALL patients with an MLL-AF4 gene rearrangement, a high level of FLT3 expression was significantly correlated with a high risk of therapy failure (39). It has been reported that FLT3 may collaborate with MLL gene fusions to promote leukemogenesis of B-ALL (65). IMC-EB10, an anti-FLT3 monoclonal antibody, can inhibit FLT3 signaling as well as significantly prolongs survival and decrease the engraftment of NOD/SCID mice injected with human B-ALL cells, indicating an important role of FLT3 in the pathogenesis of B-ALL (67). FLT3 was also demonstrated to potently activate the STAT5A in both B-ALL and AML cell lines (65). Recently, STAT5A has been shown to bind to the 3' super-enhancer of MYC and recruited BRD2 proteins to induce MYC gene expression (59), which establish a regulatory circuit that FLT3 may regulate MYC expression via activating the STAT5A. In our sub-network, upregulation of MYC expression may further promote the expression of FLT3 by the MYC/miR-15a-5p/FLT3 FFL, implying a positive feedback between MYC and FLT3 which will be involved in the pathogenesis of B-ALL. In the discussion above, we present a model of MYC/miR-15a-5p/ FLT3 FFL involving in Jak-STAT signaling pathway and regulatory network in B-ALL (Fig. 7). Based on the literature review and our sub-network analysis, we propose that MYC/ miR-15a-5p/FLT3 FFL is a potential key motif in the B-ALL miRNA-TF regulatory network, which may be associated with the occurrence and development of B-ALL.

In conclusion, the miRNA and TF-based network analysis allows us to acquire an overall view of the molecular regulatory network involved in B-ALL. It also identified some key regulators and regulatory motifs for B-ALL, as well as provided new clues for deciphering the B-ALL pathogenesis at the transcriptional and post-transcriptional levels.

\section{Acknowledgements}

This study was financially supported by the Guangdong Provincial Natural Science Foundation of China (no. 2016A030313677), the Medical Science and Technology Research Fund of Guangdong Province (no. A2016193) and the Open Project Program of Guangdong Provincial Key Laboratory of Medical Molecular Diagnostics (no. FZZD201604). We sincerely thank all donors for taking part in this investigation. 


\section{References}

1. Purizaca J, Meza I and Pelayo R: Early lymphoid development and microenvironmental cues in B-cell acute lymphoblastic leukemia. Arch Med Res 43: 89-101, 2012.

2. Sanjuan-Pla A, Bueno C, Prieto C, Acha P, Stam RW, Marschalek R and Menéndez P: Revisiting the biology of infant $\mathrm{t}(4 ; 11) / \mathrm{MLL}_{-} \mathrm{AF} 4^{+} \mathrm{B}$-cell acute lymphoblastic leukemia. Blood 126: 2676-2685, 2015.

3. Korfi K, Smith M, Swan J, Somervaille TC, Dhomen N and Marais R: BIM mediates synergistic killing of B-cell acute lymphoblastic leukemia cells by BCL-2 and MEK inhibitors. Cell Death Dis 7: e2177, 2016.

4. Luna-Aguirre CM, de la Luz Martinez-Fierro M, Mar-Aguilar F, Garza-Veloz I, Treviño-Alvarado V, Rojas-Martinez A, Jaime-Perez JC, Malagon-Santiago GI, Gutierrez-Aguirre $\mathrm{CH}$, Gonzalez-Llano $\mathrm{O}$, et al: Circulating microRNA expression profile in B-cell acute lymphoblastic leukemia. Cancer Biomark 15: 299-310, 2015.

5. Tasian SK and Gardner RA: CD19-redirected chimeric antigen receptor-modified T cells: A promising immunotherapy for children and adults with B-cell acute lymphoblastic leukemia (ALL). Ther Adv Hematol 6: 228-241, 2015.

6. Woo JS, Alberti MO and Tirado CA: Childhood B-acute lymphoblastic leukemia: A genetic update. Exp Hematol Oncol 3: 16, 2014.

7. Zhou Y, You MJ, Young KH, Lin P, Lu G, Medeiros LJ and Bueso-Ramos CE: Advances in the molecular pathobiology of B-lymphoblastic leukemia. Hum Pathol 43: 1347-1362, 2012.

8. Pegram HJ, Smith EL, Rafig S and Brentjens RJ: CAR therapy for hematological cancers: Can success seen in the treatment of B-cell acute lymphoblastic leukemia be applied to other hematological malignancies? Immunotherapy 7: 545-561, 2015.

9. Zhang XH, Wang CC, Jiang Q, Yang SM, Jiang H, Lu J, Wang QM, Feng FE, Zhu XL, Zhao T, et al: ADAM28 overexpression regulated via the PI3K/Akt pathway is associated with relapse in de novo adult B-cell acute lymphoblastic leukemia. Leuk Res 39: 1229-1238, 2015.

10. Sikaria S, Aldoss I and Akhtari M: Monoclonal antibodies and immune therapies for adult precursor B-acute lymphoblastic leukemia. Immunol Lett 172: 113-123, 2016.

11. Mullighan CG: Molecular genetics of B-precursor acute lymphoblastic leukemia. J Clin Invest 122: 3407-3415, 2012.

12. Othman MA, Grygalewicz B, Pienkowska-Grela B, Rygier J, Ejduk A, Rincic M, Melo JB, Carreira IM, Meyer B and Liehr T: A novel IGH ${ }^{@}$ gene rearrangement associated with CDKN2A/B deletion in young adult B-cell acute lymphoblastic leukemia Oncol Lett 11: 2117-2122, 2016.

13. Iacobucci I, Iraci N, Messina M, Lonetti A, Chiaretti S, Valli E, Ferrari A, Papayannidis C, Paoloni F, Vitale A, et al: IKAROS deletions dictate a unique gene expression signature in patients with adult B-cell acute lymphoblastic leukemia. PLoS One 7: e40934, 2012

14. Moorman AV: The clinical relevance of chromosomal and genomic abnormalities in B-cell precursor acute lymphoblastic leukaemia. Blood Rev 26: 123-135, 2012.

15. Yoda A, Yoda Y, Chiaretti S, Bar-Natan M, Mani K, Rodig SJ, West N, Xiao Y, Brown JR, Mitsiades C, et al: Functional screening identifies CRLF2 in precursor B-cell acute lymphoblastic leukemia. Proc Natl Acad Sci USA 107: 252-257, 2010.

16. Luan C, Yang $Z$ and Chen B: The functional role of microRNA in acute lymphoblastic leukemia: Relevance for diagnosis, differential diagnosis, prognosis, and therapy. Onco Targets Ther 8 : 2903-2914, 2015.

17. Cocco C and Airoldi I: Cytokines and microRNA in pediatric B-acute lymphoblastic leukemia. Cytokine Growth Factor Rev 22: 149-156, 2011.

18. Bousquet M, Harris MH, Zhou B and Lodish HF: MicroRNA miR-125b causes leukemia. Proc Natl Acad Sci USA 107: 21558-21563, 2010.

19. Scherr M, Elder A, Battmer K, Barzan D, Bomken S, Ricke-Hoch M, Schröder A, Venturini L, Blair HJ, Vormoor J, et al: Differential expression of miR-17-92 identifies BCL2 as a therapeutic target in BCR-ABL-positive B-lineage acute lymphoblastic leukemia. Leukemia 28: 554-565, 2014.

20. Piriyapongsa J, Jordan IK, Conley AB, Ronan $\mathrm{T}$ and Smalheiser NR: Transcription factor binding sites are highly enriched within microRNA precursor sequences. Biol Direct 6: $61,2011$.
21. Zhang HM, Kuang S, Xiong X, Gao T, Liu C and Guo AY: Transcription factor and microRNA co-regulatory loops: Important regulatory motifs in biological processes and diseases. Brief Bioinform 16: 45-58, 2015.

22. Bhatia S, Kaul D and Varma N: Potential tumor suppressive function of miR-196b in B-cell lineage acute lymphoblastic leukemia. Mol Cell Biochem 340: 97-106, 2010.

23. Ye H, Liu X, Lv M, Wu Y, Kuang S, Gong J, Yuan P, Zhong Z, Li Q, Jia H, et al: MicroRNA and transcription factor co-regulatory network analysis reveals miR-19 inhibits CYLD in T-cell acute lymphoblastic leukemia. Nucleic Acids Res 40: 5201-5214, 2012.

24. Vardiman JW, Thiele J, Arber DA, Brunning RD, Borowitz MJ, Porwit A, Harris NL, Le Beau MM, Hellström-Lindberg E, Tefferi A, et al: The 2008 revision of the World Health Organization (WHO) classification of myeloid neoplasms and acute leukemia: Rationale and important changes. Blood 114: 937-951, 2009.

25. Alvarnas JC, Brown PA, Aoun P, Ballen KK, Bellam N, Blum W, Boyer MW, Carraway HE, Coccia PF, Coutre SE, et al; Nationa Comprehensive Cancer Network: Acute lymphoblastic leukemia. J Natl Compr Canc Netw 10: 858-914, 2012.

26. Allen A, Gill K, Hoehn D, Sulis M, Bhagat G and Alobeid B: $\mathrm{C}$-myc protein expression in B-cell acute lymphoblastic leukemia, prognostic significance? Leuk Res 38: 1061-1066, 2014.

27. Pfaffl MW: A new mathematical model for relative quantification in real-time RT-PCR. Nucleic Acids Res 29: e45, 2001.

28. Sui W, Lin H, Peng W, Huang Y, Chen J, Zhang Y and Dai Y: Molecular dysfunctions in acute rejection after renal transplantation revealed by integrated analysis of transcription factor, microRNA and long noncoding RNA. Genomics 102: 310-322, 2013.

29. Dong M, Wang X, Zhao HL, Chen XL, Yuan JH, Guo JY, Li KQ and $\mathrm{Li}$ G: Integrated analysis of transcription factor, microRNA and LncRNA in an animal model of obliterative bronchiolitis. Int J Clin Exp Pathol 8: 7050-7058, 2015.

30. Lin Y, Wu J, Chen H, Mao Y, Liu Y, Mao Q, Yang K, Zheng X and Xie L: Cyclin-dependent kinase 4 is a novel target in micoRNA-195-mediated cell cycle arrest in bladder cancer cells. FEBS Lett 586: 442-447, 2012.

31. Rappaport N, Nativ N, Stelzer G, Twik M, Guan-Golan Y, Stein TI, Bahir I, Belinky F, Morrey CP, Safran M, et al: MalaCards: An integrated compendium for diseases and their annotation. Database (Oxford) 2013: bat018, 2013.

32. Felice B, Cattoglio C, Cittaro D, Testa A, Miccio A, Ferrari G, Luzi L, Recchia A and Mavilio F: Transcription factor binding sites are genetic determinants of retroviral integration in the human genome. PLoS One 4: e4571, 2009.

33. Bang SY, Kim JH, Lee PY, Chi SW, Cho S, Yi GS, Myung PK, Park BC, Bae KH and Park SG: Candidate target genes for the Saccharomyces cerevisiae transcription factor, Yap2. Folia Microbiol (Praha) 58: 403-408, 2013.

34. Lin XC, Xu Y, Sun GP, Wen JL, Li N, Zhang YM, Yang ZG, Zhang HT and Dai Y: Molecular dysfunctions in acute myeloid leukemia revealed by integrated analysis of microRNA and transcription factor. Int J Oncol 48: 2367-2380, 2016.

35. Alexiou P, Vergoulis T, Gleditzsch M, Prekas G, Dalamagas T, Megraw M, Grosse I, Sellis T and Hatzigeorgiou AG: miRGen 2.0: A database of microRNA genomic information and regulation. Nucleic Acids Res 38: D137-D141, 2010.

36. Harris MA, Clark J, Ireland A, Lomax J, Ashburner M, Foulger R, Eilbeck K, Lewis S, Marshall B, Mungall C, et al; Gene Ontology Consortium: The Gene Ontology (GO) database and informatics resource. Nucleic Acids Res 32: D258-D261, 2004.

37. Kanehisa M, Araki M, Goto S, Hattori M, Hirakawa M, Itoh M, Katayama T, Kawashima S, Okuda S, Tokimatsu T, et al: KEGG for linking genomes to life and the environment. Nucleic Acids Res 36: D480-D484, 2008.

38. Arora S, Rana R, Chhabra A, Jaiswal A and Rani V: miRNAtranscription factor interactions: A combinatorial regulation of gene expression. Mol Genet Genomics 288: 77-87, 2013

39. Zhou Y, Kanagal-Shamanna R, Zuo Z, Tang G, Medeiros LJ and Bueso-Ramos CE: Advances in B-lymphoblastic leukemia: Cytogenetic and genomic lesions. Ann Diagn Pathol 23: 43-50, 2016.

40. Brondfield S, Umesh S, Corella A, Zuber J, Rappaport AR, Gaillard C, Lowe SW, Goga A and Kogan SC: Direct and indirect targeting of MYC to treat acute myeloid leukemia. Cancer Chemother Pharmacol 76: 35-46, 2015.

41. Malin S, McManus S and Busslinger M: STAT5 in B cell development and leukemia. Curr Opin Immunol 22: 168-176, 2010 
42. Berger A, Sexl V, Valent P and Moriggl R: Inhibition of STAT5: A therapeutic option in BCR-ABL1-driven leukemia. Oncotarget 5: 9564-9576, 2014.

43. Chae HD, Mitton B, Lacayo NJ and Sakamoto KM: Replication factor $\mathrm{C} 3$ is a CREB target gene that regulates cell cycle progression through the modulation of chromatin loading of PCNA. Leukemia 29: 1379-1389, 2015.

44. Köhrer S, Havranek O, Seyfried F, Hurtz C, Coffey GP, Kim E, Ten Hacken E, Jäger U, Vanura K, O'Brien S, et al: Pre-BCR signaling in precursor B-cell acute lymphoblastic leukemia regulates PI3K/AKT, FOXO1 and MYC, and can be targeted by SYK inhibition. Leukemia 30: 1246-1254, 2016.

45. Coomans de Brachène $A$ and Demoulin JB: FOXO transcription factors in cancer development and therapy. Cell Mol Life Sci 73 $1159-1172,2016$.

46. Grosjean-Raillard J, Adès L, Boehrer S, Tailler M, Fabre C, Braun T, De Botton S, Israel A, Fenaux P and Kroemer G: Flt3 receptor inhibition reduces constitutive NFkappaB activation in high-risk myelodysplastic syndrome and acute myeloid leukemia. Apoptosis 13: 1148-1161, 2008.

47. Vacca A, Felli MP, Palermo R, Di Mario G, Calce A Di Giovine M, Frati L, Gulino A and Screpanti I: Notch3 and pre-TCR interaction unveils distinct NF-kappaB pathways in T-cell development and leukemia. EMBO J 25: 1000-1008, 2006.

48. Bruns HA and Kaplan MH: The role of constitutively active Stat6 in leukemia and lymphoma. Crit Rev Oncol Hematol 57: 245-253, 2006

49. LeBrun DP: E2A basic helix-loop-helix transcription factors in human leukemia. Front Biosci 8: s206-s222, 2003.

50. Puissegur MP, Eichner R, Quelen C, Coyaud E, Mari B, Lebrigand K, Broccardo C, Nguyen-Khac F, Bousquet M and Brousset P: B-cell regulator of immunoglobulin heavy-chain transcription (Bright)/ARID3a is a direct target of the oncomir microRNA-125b in progenitor B-cells. Leukemia 26: 2224-2232, 2012.

51. Strasser A, Harris AW, Bath ML and Cory S: Novel primitive lymphoid tumours induced in transgenic mice by cooperation between myc and bcl-2. Nature 348: 331-333, 1990.

52. Ott CJ, Kopp N, Bird L, Paranal RM, Qi J, Bowman T, Rodig SJ, Kung AL, Bradner JE and Weinstock DM: BET bromodomain inhibition targets both c-Myc and IL7R in high-risk acute lymphoblastic leukemia. Blood 120: 2843-2852, 2012.

53. Joliot V, Cormier F, Medyouf $\mathrm{H}$, Alcalde $\mathrm{H}$ and Ghysdael $\mathrm{J}$ : Constitutive STAT5 activation specifically cooperates with the loss of p53 function in B-cell lymphomagenesis. Oncogene 25: 4573-4584, 2006.
54. Nakayama J, Yamamoto M, Hayashi K, Satoh H, Bundo K, Kubo M, Goitsuka R, Farrar MA and Kitamura D: BLNK suppresses pre-B-cell leukemogenesis through inhibition of JAK3. Blood 113: 1483-1492, 2009.

55. Cho JH, Gelinas R, Wang K, Etheridge A, Piper MG, Batte K, Dakhallah D, Price J, Bornman D, Zhang S, et al: Systems biology of interstitial lung diseases: Integration of mRNA and microRNA expression changes. BMC Med Genomics 4: 8, 2011.

56. Li H: Systems genetics in '-omics' era: Current and future development. Theory Biosci 132: 1-16, 2013.

57. Lin Y, Zhang Q, Zhang HM, Liu W, Liu CJ, Li Q and Guo AY: Transcription factor and miRNA co-regulatory network reveals shared and specific regulators in the development of B cell and T cell. Sci Rep 5: 15215, 2015.

58. Jackstadt R and Hermeking H: MicroRNAs as regulators and mediators of c-MYC function. Biochim Biophys Acta 1849: 544-553, 2015.

59. Pinz S, Unser S and Rascle A: Signal transducer and activator of transcription STAT5 is recruited to c-Myc super-enhancer. BMC Mol Biol 17: 10, 2016.

60. Han SS, Han S and Kamberos NL: Piperlongumine inhibits the proliferation and survival of B-cell acute lymphoblastic leukemia cell lines irrespective of glucocorticoid resistance. Biochem Biophys Res Commun 452: 669-675, 2014.

61. Schepers H, Wierenga AT, Vellenga E and Schuringa JJ: STAT5mediated self-renewal of normal hematopoietic and leukemic stem cells. JAK-STAT 1: 13-22, 2012.

62. Tsuruyama T, Nakamura T, Jin G, Ozeki M, Yamada Y and Hiai H: Constitutive activation of Stat5a by retrovirus integration in early pre-B lymphomas of SL/Kh strain mice. Proc Natl Acad Sci USA 99: 8253-8258, 2002.

63. Gao SM, Yang J, Chen C, Zhang S, Xing CY, Li H, Wu J and Jiang L: miR-15a/16-1 enhances retinoic acid-mediated differentiation of leukemic cells and is upregulated by retinoic acid. Leuk Lymphoma 52: 2365-2371, 2011.

64. Huang E, Liu R and Chu Y: miRNA-15a/16: As tumor suppressors and more. Future Oncol 11: 2351-2363, 2015.

65. Gu TL, Nardone J, Wang Y, Loriaux M, Villén J, Beausoleil S, Tucker M, Kornhauser J, Ren J, MacNeill J, et al: Survey of activated FLT3 signaling in leukemia. PLoS One 6: e19169, 2011.

66. Annesley CE and Brown P: The biology and targeting of FLT3 in pediatric leukemia. Front Oncol 4: 263, 2014.

67. Small D: Targeting FLT3 for the treatment of leukemia. Semin Hematol 45 (Suppl 2): S17-S21, 2008. 